D. FRIEDEN ${ }^{1}$

V. $\operatorname{SICK}^{1, \infty}$

J. GRONKI ${ }^{2}$

C. SCHULZ ${ }^{2}$

\section{Quantitative oxygen imaging in an engine}

${ }^{1}$ The University of Michigan, Department of Mechanical Engineering, 2202 GG Brown Building, 2350 Hayward Street, Ann Arbor, MI 48109-2125, USA

2 Physikalisch-Chemisches Institut, Universität Heidelberg, Im Neuenheimer Feld 229, 69120 Heidelberg, Germany

Received: 4 June 2002

Published online: 8 August 2002 • ๑ Springer-Verlag 2002

ABSTRACT Simultaneous imaging of laser-induced fluorescence of toluene and 3-pentanone was used to determine the local absolute oxygen and residual gas concentrations present within an engine. The technique utilizes the different sensitivities of the laser-excited molecules to quenching by molecular oxygen as a means to determine quantitative images of in-cylinder oxygen concentrations. The difference in the amount of oxygen available between two operating conditions was investigated. Results are in agreement with measurements in the exhaust gas.

PACS 32.50.+d; 42.62.-b; 89.20.+a

1

\section{Introduction}

New engine concepts such as the spark-ignition direct-injection (SIDI) engine and the homogeneouscharge compression-ignition (HCCI) engine offer the potential for a significantly enhanced fuel economy and a drastic reduction in $\mathrm{NO}_{x}$ emissions. Studies of fuel/air mixing using laserbased imaging diagnostics have been a valuable tool in recent years for the development of such engines. Typically, a non-fluorescing base fuel (iso-octane or n-heptane) is mixed with one of several tracers that allows for laser-induced fluorescence (LIF) imaging.

3-pentanone is often used as the tracer; its fluorescence signal can be calibrated to the absolute fuel number density within the engine. In cases where no residual (burnt) gases are present, local air and hence oxygen concentrations can be calculated from the fuel concentration and the total pressure. Therefore, the fuel number density image can be converted to an equivalence ratio image $[1,2]$.
Alternatively, toluene can be used as the fluorescence tracer to measure the local equivalence ratio, as its LIF signal depends on the ratio of tracer and oxygen concentration due to severe quenching of toluene fluorescence by oxygen $[3,4]$. In the absence of residual gases, where evaporated fuel and air (with known, homogeneous oxygen concentration and total pressure) are the only compounds, the local fuel number density can be computed from the measured equivalence ratio.

Thus, in the absence of residual gases (and with knowledge of the incylinder pressure), either tracer, by itself, can be used to fully characterize the gas composition within the engine. Residual burnt gases with reduced oxygen concentration, however, "dilute" the air and cause reduced and potentially spatially varying oxygen concentrations in the endgas (i.e. the fresh air/residual gas mixture). The fuel concentrations derived from the 3-pentanone signal give no information about the local oxygen concentrations and the equivalence ratio can no longer be quantified. The toluene signal, in contrast, fails to provide any information about the absolute quantities of fuel and oxygen present. Therefore, neither tracer by itself has the ability to fully characterize the gas composition within the engine when residual gases are present.

Because residual gases are present in both SIDI and HCCI engines, and since high concentrations of recycled exhaust gases are often used to dilute the fuelair mixture (especially for HCCI operation) a technique is needed to determine the fuel and oxygen concentrations under these conditions. The fact that the 3-pentanone signal yields the fuel concentration, while the toluene signal yields the equivalence ratio hints that if the two tracers could be detected simultaneously, absolute concentrations of both fuel and oxygen could be obtained. Fortunately, since the two tracers produce spectrally separate signals, have comparable evaporative and transport properties, and since the presence of one tracer does not significantly affect the fluorescence of the other (for conditions found within the engine environment), the two tracers can indeed be used simultaneously to fully characterize the gas composition.

The two-tracer technique, which allows for the simultaneous detection of both fuel and oxygen concentrations, has been demonstrated by Koban et al. [5] in a mixing experiment of two gas jets with different compositions under room-temperature and ambientpressure conditions. The present study extends the application of this approach to the high-temperature and high-pressure conditions in the compression stroke of an internal combustion engine. It is demonstrated that the concentration 
distribution of oxygen can be measured quantitatively under engine conditions. In turn, this technique provides information about the distribution of residual gas concentrations via a balance of total in-cylinder gas amounts.

\section{2}

\section{Experimental set-up}

The measurements were conducted in a single-cylinder optical SIDI engine that featured a transparent cylinder liner, piston window (Bowditch arrangement) and windows for viewing the pent roof volume of the combustion chamber. Toluene (3\% by volume) and 3 -pentanone (15\%) were mixed with the iso-octane base fuel. This fuel mixture was directly injected into the cylinder at $270^{\circ}$ before top dead center (TDC) in an amount to yield stoichiometric combustion conditions for skip-fired operation. The spark event, when present, occurred at $20^{\circ}$ before TDC. The engine speed was held constant at $600 \mathrm{rpm}$ with a hydraulic dynamometer system. A $\mathrm{KrF}$ excimer laser (Lambda Physik EMG $150 \mathrm{~T}$ ) was chosen to excite toluene and 3 -pentanone simultaneously at $248 \mathrm{~nm}$. The laser beam was compressed to a horizontal sheet $(0.8 \mathrm{~mm}$ thick, $30 \mathrm{~mm}$ wide) with a typical pulse energy of $30 \mathrm{~mJ}$ before entering the engine at freely selectable heights (see Fig. 1).

A $50 \%$ beam splitter coated for $300 \pm 50 \mathrm{~nm}$ (Laseroptik) was used to split the signals, which were then filtered to extract the toluene fluorescence via a tunable bandpass filter

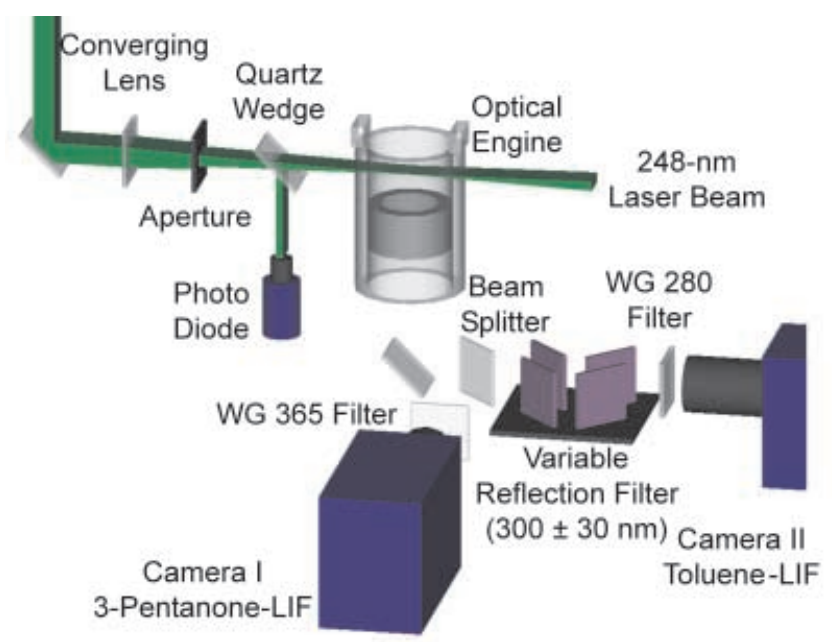

FIGURE 1 Schematic of the experimental set-up. After splitting with a $300 \pm 50 \mathrm{~nm} 50 \%$ beam splitter, the signals were filtered to isolate 3-pentanone and toluene LIF signals for recording with separate ICCD cameras
(Laseroptik) set to $300 \pm 30 \mathrm{~nm}$ and the 3-pentanone signal via a WG365 Schott long-pass filter. The fluorescence signals were imaged onto two intensified CCD cameras (LaVision FlameStar II). The laser pulse energy was recorded along with the full pressure trace of each engine cycle for which fluorescence images were taken. These data were fed into the camera host computer via an additional A/D converter unit and stored along with the images for further processing.

\section{Theoretical background and data analysis}

For weak laser excitation, the LIF signal intensity, $S_{\mathrm{LIF}}$, is given by:

$$
\begin{aligned}
S_{\mathrm{LIF}}= & c I_{\mathrm{Laser}}(\lambda) V N \sigma(\lambda, p, T, y) \\
& \times \phi(\lambda, p, T, y),
\end{aligned}
$$

where $I_{\text {Laser }}$ is the local laser energy density in the detection volume $V, N$ is the number density, $\sigma$ the molecular absorption cross section and $\phi$ the fluorescence quantum yield of the tracer molecules. The absorption cross section and fluorescence quantum yield depend on the excitation wavelength $\lambda$, pressure $p$, temperature $T$, and mixture composition $y$. The factor $c$ comprises geometrical arrangements and detection optics properties.

The dependence of 3-pentanone LIF on temperature, pressure and gas composition has been studied in the past [6-8], and raw signals can be corrected using this information provided the pressure and temperature are known. Similar dependencies for toluene, in contrast, have not yet been completely characterized. For toluene, the fluorescence quantum yield $\phi$ is strongly reduced by collisional fluorescence quenching by molecular oxygen (2). Quenching depopulates the excited state with the quenching rate coefficient $k_{\mathrm{q}, \mathrm{O}_{2}}$ proportional to the oxygen partial pressure $p_{\mathrm{O}_{2}}$.

$\phi \propto \frac{1}{k_{f}+k_{\mathrm{q}, \mathrm{O}_{2}} p_{\mathrm{O}_{2}}}$

As long as this oxygen quenching rate is fast compared to the relaxation of the excited states through other channels $\left(k_{f}\right)$, the toluene fluorescence decreases inversely proportional to oxygen partial pressure. At low oxygen concentrations, where the alternative depopulation channels can no longer be neglected, the fluorescence intensity in the absence of oxygen $I_{\text {toluene-LIF }}^{0}$ relative to the fluorescence intensity in the presence of oxygen $I_{\text {toluene-LIF }}$ follow the Stern-Volmer relation (3) with the Stern-Volmer coefficient $k_{\mathrm{SV}}=k_{\mathrm{q}} / k_{f}$.

$I_{\text {toluene-LIF }}^{0} / I_{\text {toluene-LIF }} \propto 1+k_{\mathrm{SV}} p_{\mathrm{O}_{2}}$

Previous work $[3,4]$ on toluene fluorescence excited at $248 \mathrm{~nm}$ has demonstrated that for near-stoichiometric conditions, like in homogeneously mixed combustion engines, the signals are proportional to the toluene number density and inversely proportional to the number density of molecular oxygen. Other colliders, like water, carbon dioxide and nitrogen, have no significant quenching influence [3]. This indicates that $k_{f}$ will not be important for the conditions present in the engine of this study, i.e. under near-stoichiometric operation. Furthermore, Koban et al. [5] point out that toluene can be effectively quenched by 3-pentanone. For the gas composition in the present study (oxygen : fuel : tracer $\sim 25: 5: 1$ ), oxygen will still dominate toluene quenching. The measured LIF signal is thus a function of the ratio of toluene and oxygen, or alternatively, the equivalence ratio:

$$
\begin{aligned}
S_{\text {Toluene }} & \propto \frac{[\text { Toluene }]}{[\text { Oxygen }]} \propto \frac{[\text { Fuel }]}{[\text { Oxygen }]} \\
& \propto \text { Equivalence ratio } .
\end{aligned}
$$


In fuel-rich areas like in direct-injecting engines, where the assumption of $k_{f} \ll$ $k_{\mathrm{p}} p_{\mathrm{O}_{2}}$ is no longer valid, data evaluation based on (3) still allows the evaluation of equivalence ratios from tolueneLIF intensities as long as the SternVolmer factor is known. By comparing (1) to (4), it can be seen that the "constant" of proportionality in (4), and thus the toluene fluorescence signal, could possibly depend on both temperature and pressure. Reboux et al. [3] have demonstrated that the signal is insensitive to variations in pressure at pressures above 3 bar. Thus, at the pressures found within the engine near TDC, the toluene signal depends on (and must be corrected for) temperature only. The 3-pentanone signal, in contrast, must be corrected for both temperature and pressure effects.

By motoring the engine without igniting the injected fuel, the dependence of the PLIF signals on crank-angle (i.e. the dependence on both pressure and temperature combined) can be ascertained. This is shown in Fig. 2 for both tracers. The variation of the toluene LIF intensity is due to changes in temperature only; the starting temperature is known at the time of the intake valve closing (IVC), and the asymmetric nature of the curve about TDC indicates that significant heat loss is occurring. Also shown in Fig. 2 is the crank-angle dependence of the 3-pentanone signal, which has a more complicated form as it is sensitive to both pressure and temperature. Its unique shape is attributed to both heat transfer losses and gas losses due to piston-ring blow-by (which is significant in the optical engine and only affects the 3-pentanone signal). With the two curves of Fig. 2, the measured pressure trace, the measured temperature at IVC, the ideal gas law and the known temperature and pressure dependence of 3-pentanone, an iterative calculation was performed to determine the three pieces of information needed to correct raw toluene and 3-pentanone signals for the given engine conditions: 1) the in-cylinder temperature; 2) the trapped mass within the cylinder (which varies with crank-angle); and 3) the temperature dependence of toluene LIF signals.

Since the imaged region in the cylinder was at least $10 \mathrm{~mm}$ away from the walls, the assumption of a homogeneous bulk temperature was justified [2]. The raw LIF images could then be corrected
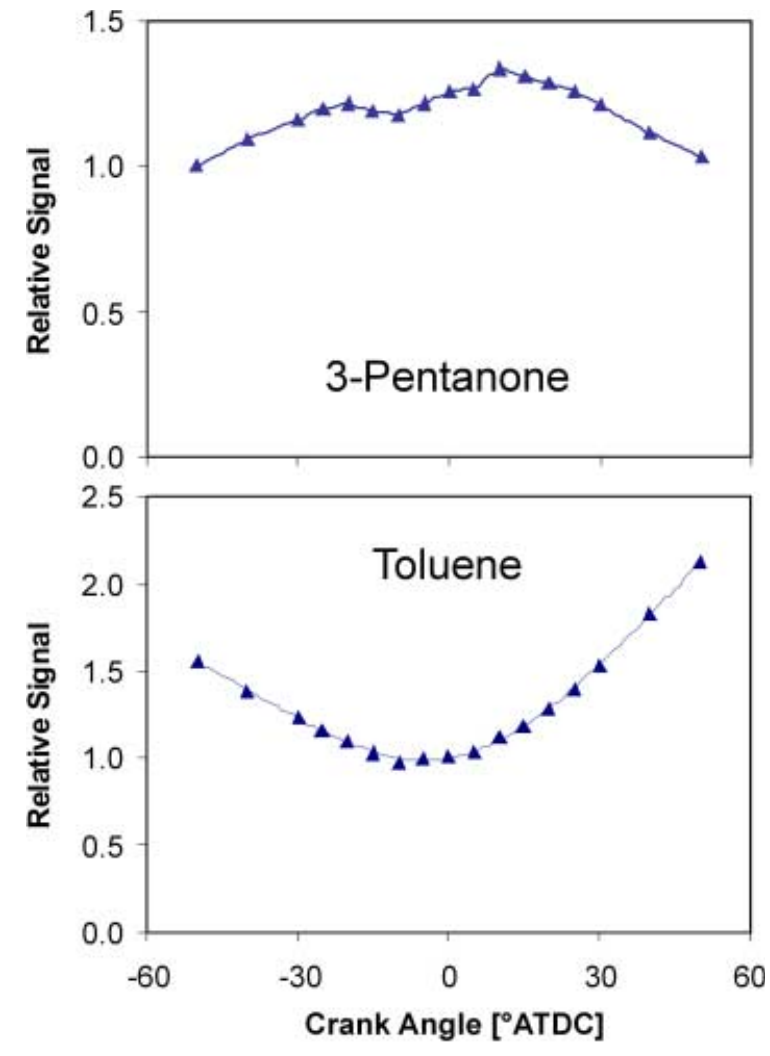

FIGURE 2 Measured LIF signal as a function of engine crank-angle for 3-pentanone and to yield the local fuel concentrations (3-pentanone) and equivalence ratios (toluene). The local oxygen concentrations were then computed from the ratio of fuel concentration and equivalence ratio, i.e. the corrected 3-pentanone signal divided by the corrected toluene LIF signal. If spatial temperature gradients existed, a simultaneous measurement of temperature would be needed to account for the then locally different correction factors to account for the temperaturesensitivity of the tracers.

In order to demonstrate the oxygen imaging technique, two different operating conditions were examined. For the first case, the engine was operated under skip-fired conditions; the fuel was only injected and ignited every sixth cycle. This ensured that no burnt gases were left in the cylinder. For the second case, the fuel was injected and ignited every single cycle. For this continuous-fired mode, a certain amount of burnt gases from the previous cycle will remain in the cylinder. It was thus expected that reduced oxygen concentrations would be present for continuous-fired operation, since residual exhaust gases were now present instead of the "residual air" in the skip-fired case. Since the amount of fuel injected between the two cases was kept constant, the decreased oxygen concentration in the continuous-fired case should be reflected in the equivalence ratio measurement (toluene signal), and ultimately in the calculation of oxygen concentration results. Since the combustion, and hence the cylinder pressure, varied from cycle to cycle, the images were processed prior to any averaging, as pressure and temperature are non-linearly coupled and also affect the LIF signals in a non-linear manner.

Figure 3 shows corrected images of the in-cylinder oxygen distribution as a function of crank-angle for both skip-fired and continuous-fired operation. The images were taken near the spark plug for crank angles up to TDC. Close to TDC, the flame has consumed all of the fuel (and with that the tracer) in the viewable area and thus, LIF signals can no longer be obtained.

The oxygen concentration distributions of Fig. 3 are spatially quite homogeneous but show significant differences in magnitude between the two operating conditions. This is better seen 


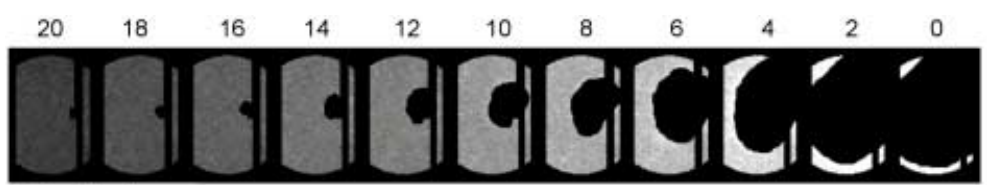

Skip-fired

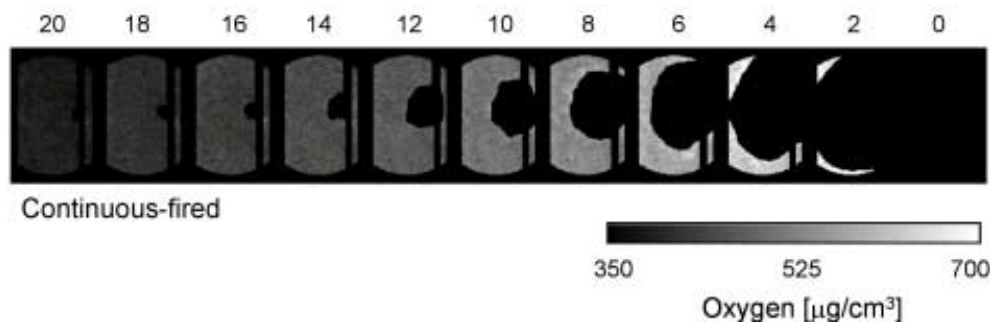

FIGURE 3 Oxygen concentration distribution in a horizontal plane near the spark plug (the black vertical stripe in the images is due to an intentional blockage of the laser beam to prevent it from scattering off the spark plug). The numbers in the images denote the crank-angle position before top dead center (TDC). The upper row shows results from skip-injected/fired (every sixth cycle) engine operation; the lower row shows the oxygen concentration for continuous injection and firing. Note that the flame also burns faster in the continuous-fired mode

in the graphs of Fig. 4. Derived from the 3-pentanone signals, the in-cylinder fuel concentration was the same for both cases (the same amount of fuel was injected). The equivalence ratio, in contrast, was higher for continuousfired operation as less oxygen was available in this mode; the equivalence ratio increased from 1.0 to 1.1 in going from skip- to continuous-fired operation. This is in excellent agreement with measurements in the exhaust gas using a standard lambda meter (ETAS). Also, the geometric properties of the engine were such that the clearance volume at TDC was about $10 \%$ of the maximum volume. This was approximately the amount of residual burnt gas that then was expected to remain in the cylinder between cycles in a continuous-fired operating mode.

For the purpose of comparison, the spatially averaged data for the case where the engine was only motored, i.e. fuel is injected but not ignited, is also shown. It is apparent that beyond about $10^{\circ}$ before TDC, the fuel and oxygen concentrations of the end-gas region increase beyond the concentrations of the motored case. This increase in number density was expected because of the higher pressures associated with the firing condition. Furthermore, this deviation of the data in the fired and the motored case occurred within a few crank-angle degrees of a similar deviation in pressure traces (not shown). Note that the equivalence ratio remained unchanged beyond $10^{\circ}$ before TDC. The
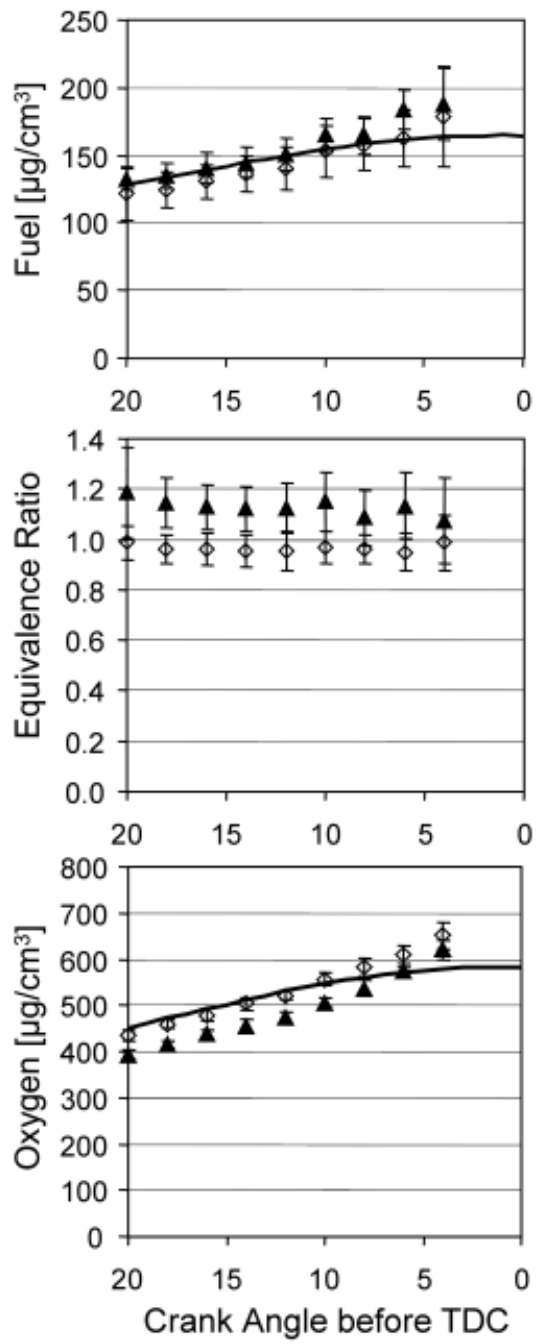

FIGURE 4 Fuel concentration, equivalence ratio and oxygen concentration for motored, skip-fired (every sixth cycle) and continuous-fired engine operation. Error bars indicate $\pm 2 \sigma$ of the spatial variation of the data. (---, motored; $\diamond$ skip-fired; $\Delta$ continuously-fired) data in Fig. 4 are spatial averages; the error bars show the $2 \sigma(95 \%)$ interval of the spatial variation of the fuel and oxygen concentrations as well as that of the equivalence ratio. Data from different areas of the image were taken for this purpose.

The amount of oxygen measured in the skip-fired case was essentially the same as for the motored (no-combustion) case. This indicates that within five cycles, where no fuel is injected and no spark is fired, all of the burnt gases are removed, no residual gas remains in the cylinder and only fresh air and fuel are present in the sixth cycle when ignition occurs again. In contrast to this, the amount of oxygen was reduced by approximately $10 \%$ when the engine was fired every cycle. For the stoichiometric combustion conditions of the present test, it can be assumed that fuel and air completely burn and thus, the residual gas in the cylinder will not contribute to the amount of oxygen measured. Thus, residual gas makes up for the measured difference in in-cylinder volume and in turn the oxygen concentration can be used to assess the concentration of residual gases.

\section{Conclusions and future work}

A method is demonstrated for quantitative imaging of oxygen concentration distributions in engines. Simultaneous excitation with a $\mathrm{KrF}$ excimer laser at $248 \mathrm{~nm}$ and detection of spectrally separated PLIF signals of toluene and 3-pentanone, which are used as tracers in iso-octane fuel, yields information about the equivalence ratio and the absolute number density of fuel. The combination of both signals yields the oxygen concentration. For two different operating conditions of a gasoline direct-injection engine, the method provides quantitative results in agreement with metered fuel and air flows, as well as exhaust gas analysis.

Extending this method to conditions with spatial temperature gradients might require simultaneous temperature measurements to allow for proper correction of the LIF signals. This could be achieved via LIF imaging of, for example, 3-pentanone with the use of an additional excitation wavelength and detector [2]. 
The technique presented here also provides information about the amount of residual gas that is present in the cylinder via a number density balance. This is of particular interest as direct imaging of exhaust gas components like water [9] or carbon dioxide [10] does not seem feasible under engine conditions and tracer techniques for imaging residual gases in engines have not yet been fully developed [11].

ACKNOWLEDGEMENTS This research was sponsored by U.S. Department of Energy, Office of Basic Energy Sciences, Division of Chemical Sciences, Geosciences and
Biosciences, Contract No. DE-FG02-98ER14915 - Subcontract No. 14412-S1 (Dr. A. Laufer), and by General Motors Corporation through the General Motors Collaborative Research Laboratory at The University of Michigan. The National Science Foundation (Dr. M. Suskin) and DAAD are also acknowledged for their support through an international exchange program, No. INT-9815290, which allowed JG and CS to visit The University of Michigan for the experimental work.

\section{REFERENCES}

1 M. Richter, B. Axelsson, K. Nyholm, M. Aldén: Proc. Comb. Inst. 27, 51 (1998)

2 S. Einecke, C. Schulz, V. Sick: Appl. Phys. B 71, 717 (2000)
3 J. Reboux, D. Puechberty, F. Dionnet: SAE Technical Paper Series 941988 (1994)

4 H. Krämer, S. Einecke, C. Schulz, V. Sick, S.R. Nattrass, J.S. Kitching: SAE Technical Paper Series 982467 (1998)

5 W. Koban, J. Schorr, C. Schulz: Appl. Phys. B 74, 111 (2001)

6 F. Großmann, P. Monkhouse, M. Ridder, V. Sick, J. Wolfrum: Appl. Phys. B 62, 249 (1996)

7 F. Ossler, M. Aldén: Appl. Phys. B 64, 493 (1997)

8 J.D. Koch, R.K. Hanson: Appl. Opt. (2002) submitted

9 R.W. Pitz, T.S. Cheng, J.A. Wehrmeyer, C.F. Hess: Appl. Phys. B 56, 94 (1993)

10 B.J. Kirby, R.K. Hanson: Appl. Phys. B 69, 505 (1999)

11 V. Sick: Appl. Phys. B 74, 461 (2002) 